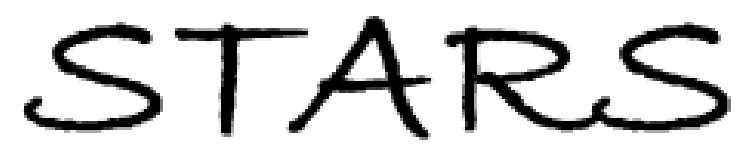

University of Central Florida

STARS

$1-1-2013$

\title{
Ultrahigh vacuum-compatible sockets for pin grid arrays used in nanoscale and atomic physics
}

Jyoti Katock

University of Central Florida

Cameron Glasscock

University of Central Florida

Masa Ishigami

University of Central Florida

Find similar works at: https://stars.library.ucf.edu/facultybib2010 University of Central Florida Libraries http://library.ucf.edu

This Article is brought to you for free and open access by the Faculty Bibliography at STARS. It has been accepted for inclusion in Faculty Bibliography 2010 s by an authorized administrator of STARS. For more information, please contact STARS@ucf.edu.

\section{Recommended Citation}

Katock, Jyoti; Glasscock, Cameron; and Ishigami, Masa, "Ultrahigh vacuum-compatible sockets for pin grid arrays used in nanoscale and atomic physics" (2013). Faculty Bibliography 2010s. 4188.

https://stars.library.ucf.edu/facultybib2010/4188

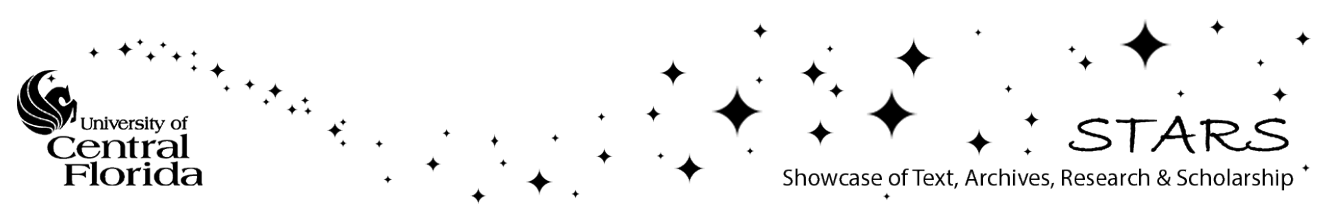




\section{Ultrahigh vacuum-compatible sockets for pin grid arrays used in nanoscale and atomic physics}

Jyoti Katoch, Cameron Glasscock, and Masa Ishigami

Citation: Journal of Vacuum Science \& Technology B 31, 023201 (2013); doi: 10.1116/1.4793278

View online: $\mathrm{https}: / /$ doi.org/10.1116/1.4793278

View Table of Contents: https://avs.scitation.org/toc/jvb/31/2

Published by the American Vacuum Society

\section{ARTICLES YOU MAY BE INTERESTED IN}

Atomic resolution scanning tunneling microscopy in a cryogen free dilution refrigerator at $15 \mathrm{mK}$

Review of Scientific Instruments 85, 035112 (2014); https://doi.org/10.1063/1.4868684

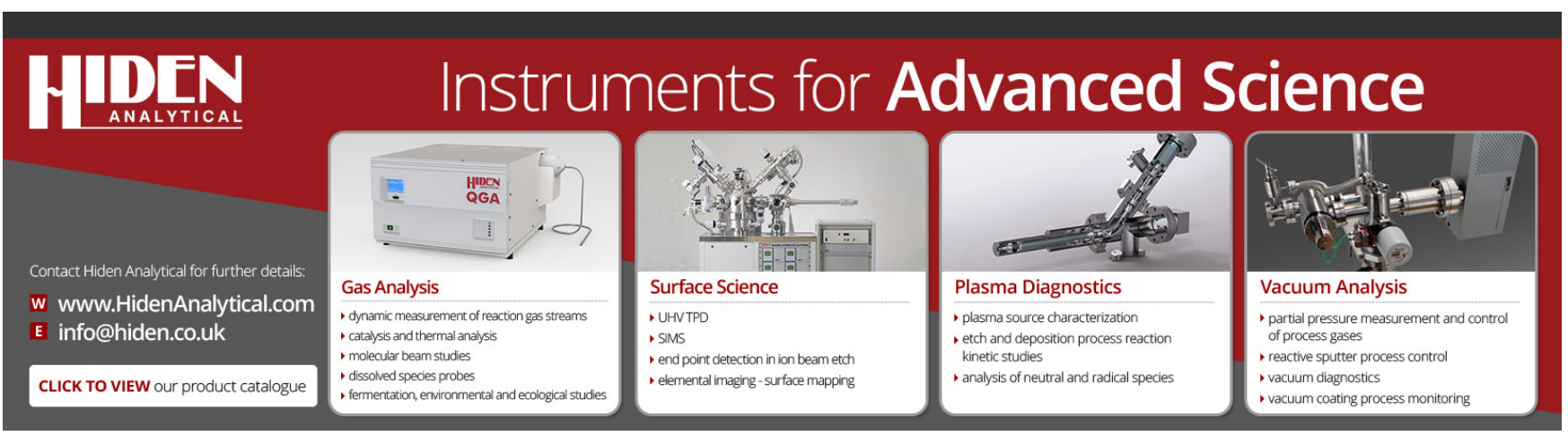




\title{
SHOP NOTES
}

These are "how to do it" papers. They should be written and illustrated so that the reader may easily follow whatever

instruction or advice is being given.

\section{Ultrahigh vacuum-compatible sockets for pin grid arrays used in nanoscale and atomic physics}

\author{
Jyoti Katoch, Cameron Glasscock, and Masa Ishigami \\ Department of Physics and Nanoscience Technology Center, University of Central Florida, \\ Orlando, Florida 32816
}

(Received 4 December 2012; accepted 4 February 2013; published 28 February 2013)

\begin{abstract}
Pin grid arrays are often used for packaging devices for nanoscale and atomic physics experiments performed in ultrahigh vacuum (UHV). These arrays require their complementary socket arrays for measurements, yet UHV-compatible sockets are not commercially available. In this paper, the authors describe the design and construction of fully UHV-compatible sockets, which provide reliable electronic contact down to cryogenic temperatures and survive repeated insertion-retraction cycles. (C) 2013 American Vacuum Society. [http://dx.doi.org/10.1116/1.4793278]
\end{abstract}

\section{INTRODUCTION}

Pin grid arrays (PGAs) are used commercially for packaging integrated circuits. Their compact geometry, large contact density, and commercial availability have also made them useful for nanoscale and atomic physics. In these fundamental science applications, it is often necessary to use ultrahigh vacuum (UHV)-compatible PGAs. Nanoscale materials such as graphene, ${ }^{1,2}$ carbon nanotubes, ${ }^{3-6}$ and nanowires ${ }^{7}$ possess extreme sensitivities to adsorbates because of their high surface to volume ratio. Such sensitivities necessitate experiments in UHV in which absolute control over adsorbates can be established. UHV transport measurements at temperatures ranging from 10 to $490 \mathrm{~K}$ have been carried out to discover the impact of adsorbates, ${ }^{8-12}$ intentionally created atomic scale defects, ${ }^{13}$ and substrate phonons ${ }^{14}$ on graphene and nanotubes. UHV-compatible PGAs are used to package nanoscale devices in these experiments. In atomic physics, there is significant interest in fabricating ion traps on chips with high spatial density ${ }^{15,16}$ for developing atomic clocks and quantum computing technologies. UHV is desired for increasing trapping times and ensuring the purity of trapped atoms and molecules in these experiments, ${ }^{17,18}$ and UHV-compatible PGAs are also used to package these on-chip ion traps. Thus, devices for both nanoscale and atomic physic necessitate UHV-compatible PGAs. Fortunately, these PGAs are readily available and can be purchased from Kyocera (San Diego, CA), Ametek (Lakewood, NJ), and other manufacturers.

Since devices are extremely sensitive and do not tolerate soldering, PGAs require their complementary socket arrays (SAs) to establish electronic contacts. Typically, a given instrument has an SA fixed to the vacuum chamber and PGAs are exchanged to measure different devices. Commercial sockets, shown in Figs. 1(a) and 1(b), can be integrated with nonplastic insulating support to assemble SAs for the use in UHV as shown in Fig. 1(c) and as demonstrated in previous studies. ${ }^{8,9,15,16}$ Yet, these SAs based on commercial sockets should not be considered to be UHV-compatible. The goldplated housing for these commercial sockets, indicated by the shaded region in Fig. 1(b), is composed of brass. Since the plating is thin and composed of a $0.25 \mu \mathrm{m}$ gold layer with a $1.25 \mu \mathrm{m}$ nickel base layer, even small scratches can cause zinc contamination detrimental to UHV as brass contains zinc. All commercial sockets use similar brass-based housing and, consequently, there are no UHV-compatible sockets available for assembling SAs.

To address this, we have designed completely UHVcompatible sockets, which maintain reliable electronic contact down to cryogenic temperatures and survive repeated insertion-retraction cycles. We describe its design and construction in detail below.

\section{DESIGN AND CONSTRUCTION OF UHV-COMPATIBLE SOCKETS AND SAS}

The cross-sectional mechanical drawing of the UHVcompatible socket is shown in Fig. 2(a). The socket has two components: (1) a gold-plated beryllium copper $(\mathrm{BeCu})$ spring contact and (2) a stainless steel tube.

A gold-plated $\mathrm{BeCu}$ spring contact, as shown in Fig. 2(b), serves as the contacting electrode in our design. Spring contacts can be extracted from Samtec SC series sockets [Fig. 1(a)]. To retrieve a spring contact, vertical cuts are made at the top of the brass housing of a Samtec socket using a wire cutter. The housing is opened and the spring contact is removed by using small tweezers.

Spring contacts are very thin and fragile. In order to ensure their survival under repeated insertion-retraction cycles, spring contacts are press-fitted into stainless steel tubes with 1/16 in. outer diameter and 0.010 in. wall thickness as shown in Fig. 2(c). Stainless steel tubes are first cut into appropriate lengths using a diamond saw and deburred with a small drill bit. Spring contacts and deburred stainless 

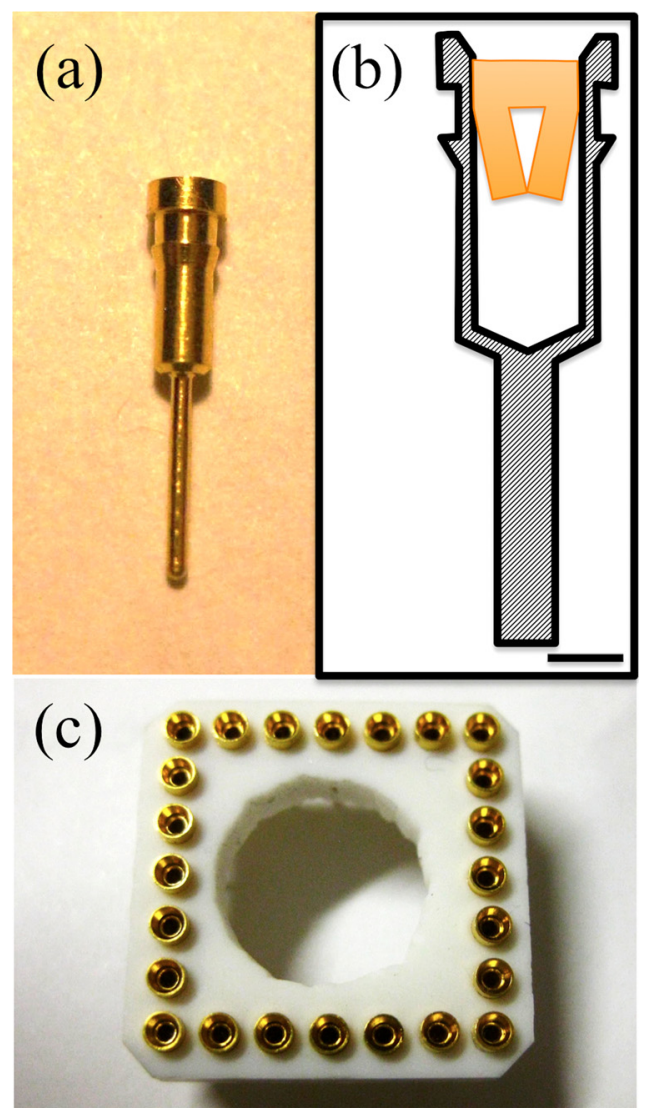

FIG. 1. (Color online) (a) Photograph of a Samtec SC series socket. (b) Schematic cross section of a Samtec SC series socket. The orange region (grey in print) denotes the gold-plated $\mathrm{BeCu}$ spring contact. The shaded region represents the gold-plated brass casing for the spring contact. The scale bar is $1 / 32$ in. (c) A socket array for a PGA fabricated from the commercial sockets. The insulating support is made from MACOR ceramic and sockets are glued using nonconductive epoxy.

tubes are cleaned using acetone and isopropanol to remove any residues before press-fitting.

Press-fitted contacts are then epoxied to tubes as shown in Fig. 2(a). We apply conductive epoxy, H20E from Epoxy
Technology, Inc (Billerica, MA), along the edge where the contact meets the steel tube. Once the conductive epoxy layer cures, nonconductive epoxy, H77 from Epoxy Technology, Inc. (Billerica, MA), is applied to increase the strength of the bond between the spring contact and the tube. These epoxies survive being baked up to $200{ }^{\circ} \mathrm{C}$ without bond failure and with less than $1 \%$ of weight loss, according to tests performed by Epoxy Technology.

Completed custom sockets are glued into a machinable ceramic plate [Macor, Corning, Inc. (Corning, NY)] with a pattern matching that of a PGA to assemble an SA as shown in Fig. 2(d). Nonconducting epoxy is used for gluing the sockets into the plate. A dummy PGA is used for the bonding procedure to ensure the proper alignment.

\section{CHARACTERISTICS}

The completed custom SA is UHV-compatible, reliable down to low temperatures, and robust under insertion-retraction cycles. We have performed transport measurements on a graphene device to demonstrate. Any adsorbates, which might be introduced by contamination, can affect the minimum voltage, $\mathrm{V}_{\text {min }}$, and the field effect mobility. ${ }^{8} \mathrm{~V}_{\min }$ is the gate voltage at which the conductivity is minimized and the mobility is proportional to the slope of the gate dependent conductivity curve.

Figure 3 shows transport measurements performed on a graphene device. The device was first introduced into vacuum and baked along with the chamber to establish UHV. Pressures reaching down to $1.8 \times 10^{-10}$ Torr can be achieved with the custom SA integrated to our UHV cryostat, which is LT-3B purchased from Advanced Research Systems, Inc. (Macungie, PA). Before baking, $\mathrm{V}_{\min }$ is located at $+2.7 \mathrm{~V}$ and after baking, during which the device reaches $380 \mathrm{~K}$, $\mathrm{V}_{\text {min }}$ shifts to $-1.7 \mathrm{~V}$. The electron mobility improves and the hole mobility is slightly decreased. The observed change in transport property is minimal and can be attributed to changes in the properties of the substrate as substrate-bound adsorbates are desorbed. If the bakeout process did introduce contamination, annealing at higher temperatures should
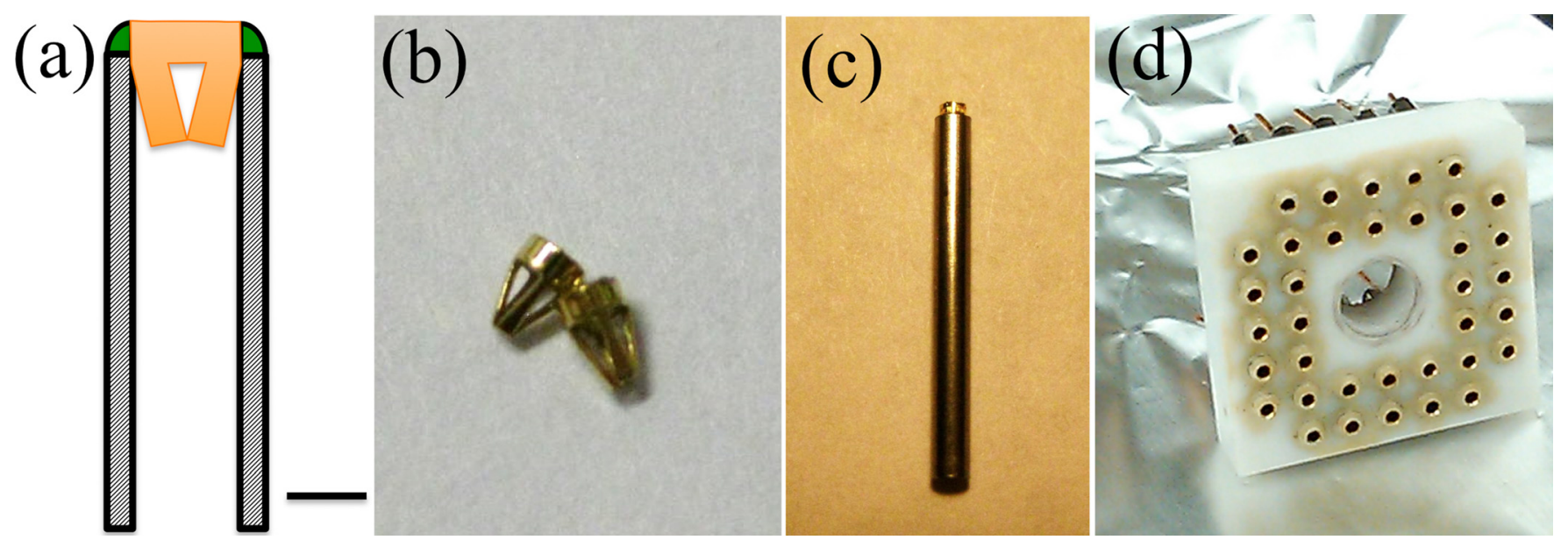

FIG. 2. (Color online) (a) Schematic cross section of the custom socket. The orange region (grey in print) represents the gold-plated BeCu spring contact. The shaded region is the stainless steel tube. The green region represents the epoxy layers. The scale bar is 1/32 in. (b) A photograph of an extracted gold-plated $\mathrm{BeCu}$ spring contact. (c) The spring contact press-fitted in a stainless steel tube. (d) A socket array for a PGA fabricated from the custom sockets. 


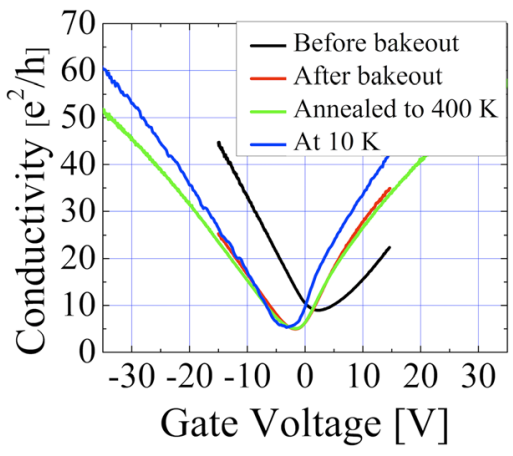

FIG. 3. (Color online) Representative transport measurement performed on a graphene device, showing that the new sockets are UHV-compatible and reliable down to $10 \mathrm{~K}$. Gate-dependent conductivity at room temperature in air, after baking, after annealing at $400 \mathrm{~K}$ is shown. In addition, gatedependent conductivity at $10 \mathrm{~K}$ is also plotted.

worsen the device. Yet, the transport property remains the same after annealing at $400 \mathrm{~K}$ indicating that there are no contaminations. As such, we conclude that the custom SA is UHV-compatible. Figure 3 also shows that the custom SA can also maintain reliable electronic contact down to $10 \mathrm{~K}$.

\section{CONCLUSION}

We have designed a new socket for PGAs that is UHVcompatible and can maintain reliable electronic contact down to $10 \mathrm{~K}$. These sockets can be used to fabricate high contact density SAs, which are useful for nanoscale and atomic physics experiments.

\section{ACKNOWLEDGMENT}

This work was based upon research supported by the National Science Foundation under Grant No. 0955625.

${ }^{1}$ A. K. Geim, Science 324, 1530 (2009).

${ }^{2}$ F. Schedin, A. K. Geim, S. V. Morozov, E. W. Hill, P. Blake, M. I. Katsnelson, and K. S. Novoselov, Nature Mater. 6, 652 (2007).

${ }^{3}$ J. C. Charlier, X. Blase, and S. Roche, Rev. Mod. Phys. 79, 677 (2007).

${ }^{4}$ H. J. Dai, Surf. Sci. 500, 218 (2002).

${ }^{5}$ H. J. Dai, Acc. Chem. Res. 35, 1035 (2002).

${ }^{6}$ D. R. Kauffman and A. Star, Angew. Chem., Int. Ed. 47, 6550 (2008).

${ }^{7}$ F. Patolsky and C. M. Lieber, Mater. Today 8, 20 (2005).

${ }^{8}$ J. H. Chen, C. Jang, S. Adam, M. S. Fuhrer, E. D. Williams, and M. Ishigami, Nat. Phys. 4, 377 (2008).

${ }^{9}$ J. Katoch, C. Chen, R. Tsuchikawa, C. W. Smith, E. R. Mucciolo, and M. Ishigami, Phys. Rev. B 82, 081417 (2010).

${ }^{10}$ K. M. McCreary, K. Pi, A. G. Swartz, W. Han, W. Bao, C. N. Lau, F. Guinea, M. I. Katsnelson, and R. K. Kawakami, Phys. Rev. B 81, 115453 (2010).

${ }^{11}$ K. M. McCreary, A. G. Swartz, W. Han, J. Fabian, and R. K. Kawakami, Phys. Rev. Lett. 109, 186604 (2012).

${ }^{12}$ K. Pi, W. Han, K. M. McCreary, A. G. Swartz, Y. Li, and R. K. Kawakami, Phys. Rev. Lett. 104, 187201 (2010).

${ }^{13}$ J. H. Chen, W. G. Cullen, C. Jang, M. S. Fuhrer, and E. D. Williams, Phys. Rev. Lett. 102, 236805 (2009).

${ }^{14}$ B. Chandra, V. Perebeinos, S. Berciaud, J. Katoch, M. Ishigami, P. Kim, T. F. Heinz, and J. Hone, Phys. Rev. Lett. 107, 146601 (2011).

${ }^{15}$ D. Stick, W. K. Hensinger, S. Olmschenk, M. J. Madsen, K. Schwab, and C. Monroe, Nat. Phys. 2, 36 (2006).

${ }^{16}$ J. J. McLoughlin, A. H. Nizamani, J. D. Siverns, R. C. Sterling, M. D. Hughes, B. Lekitsch, B. Stein, S. Weidt, and W. K. Hensinger, Phys. Rev. A 83, 013406 (2011).

${ }^{17}$ W. D. Phillips, Rev. Mod. Phys. 70, 721 (1998).

${ }^{18}$ J. Weiner, V. S. Bagnato, S. Zilio, and P. S. Julienne, Rev. Mod. Phys. 71, 1 (1999). 